\title{
BMJ Open High incidence of HIV and syphilis among migrant men who have sex with men in Beijing, China: a prospective cohort study
}

\author{
Hengyi Mao, ${ }^{1}$ Wenzhe Ma, ${ }^{1}$ Hongyan Lu, ${ }^{2}$ Lu Wang, ${ }^{3}$ Hui Zheng, ${ }^{1}$ Yingying Zhu, ${ }^{1}$ \\ Zhihang Peng, ${ }^{1}$ Rongbin Yu, ${ }^{1}$ Ning Wang ${ }^{3}$
}

To cite: Mao H, Ma W, Lu H, et al. High incidence of HIV and syphilis among migrant men who have sex with men in Beijing, China: a prospective cohort study. BMJ Open 2014;4:e005351. doi:10.1136/bmjopen-2014005351

- Prepublication history for this paper is available online. To view these files please visit the journal online (http://dx.doi.org/10.1136/ bmjopen-2014-005351).

$\mathrm{HM}$ and WM contributed equally. ZP and NW are cocorresponding authors.

Received 27 March 2014 Revised 6 August 2014 Accepted 7 August 2014

CrossMark

For numbered affiliations see end of article.

Correspondence to Dr Zhihang Peng; zhihangpeng@njmu.edn.cn and Dr Ning Wang; wangning_12@126.com

\section{ABSTRACT}

Objectives: The purpose of the study was to investigate the incidence of HIV and syphilis and their related factors, as well as to examine the predictors associated with seroconversion among migrant men who have sex with men (MSM) in Beijing, China.

Design: Prospective cohort study.

Setting: Beijing, China.

Participants: 511 HIV-negative migrant MSM.

Primary and secondary outcome measures:

Sociodemographic and behavioural information of participants, HIV and syphilis incidence rates, and factors associated with HIV/syphilis seroconversion and cohort retention.

Results: Of the 511 participants, $60.3 \%$ (308) and $52.4 \%$ (273) were retained at the 4- and 8-month follow-up visits, respectively. The HIV and syphilis incidence rates were $7.83(95 \% \mathrm{Cl} 4.48$ to 12.72$)$ and 11.11 (95\% Cl 6.47 to 17.80 ) per 100 person-years, respectively. HIV seroconversion was significantly associated with 'had first anal intercourse at age 15 or younger' (adjusted HR (aHR) 9.20, 95\% Cl 1.94 to 43.56) and 'ever had group sex' (aHR $4.30,95 \% \mathrm{Cl}$ 1.40 to 13.18). Bisexual orientation (aHR $5.09,95 \% \mathrm{Cl}$ 1.02 to 25.57 ) was significantly associated with syphilis seroconversion. Predictors associated with both 4- and 8-month retention rates included age $\geq 25$ years old and living in Beijing for more than 1 year.

Conclusions: The high incidence of HIV and syphilis among migrant MSM is a cause for concern.

A comprehensive strategy should be implemented to maintain a higher retention rate among migrant MSM.

\section{INTRODUCTION}

Over the last decade, sexual transmission has become the dominant route of HIV transmission in China. ${ }^{1}$ The percentage of cases attributed to homosexual transmission rose from $7.3 \%$ in 2005 to $16.1 \%$ in 2011 , comprising an increasing proportion of such infections among people living with HIV/AIDS

\section{Strengths and limitations of this study}

- This is the first cohort study to assess the incidence of HIV and syphilis among migrant men who have sex with men in Beijing.

- Cox regression analysis and stepwise multiple logistic regression were used to identify factors associated with HIV/syphilis seroconversion and cohort retention, respectively.

- Retention rates were relatively low, which means that the estimates of HIV/syphilis seroconversions may be biased because it is not known whether characteristics of the participants retained in the study were the same as those of the participants lost to follow-up.

- Sampling methods may have led to selection bias.

- None of the participants had migrated from Beijing, and the proportions of migrants to Beijing from different provinces varied, which may lead to difficulties with generalisation of the data.

(PLWHA). ${ }^{2}$ The 2011 HIV epidemic assessment report stated that homosexual transmissions accounted for $17.4 \%$ of infections among the estimated 780000 PLWHA, an increase from $14.7 \%$ in 2009. ${ }^{3}$ A growing number of studies have revealed an upward trend in HIV incidence among men who have sex with men (MSM) in different parts of China. For example, incidence rates per 100 person-years (PY) increased from 8.0 to 15.4 in 2006-2009 in Chongqing, from 5.0 to 6.3 in 2010-2012 in Zhejiang, ${ }^{4}$ from 1 to 10.2 in 2007-2009 in Liaoning, 5.7 to 8.2 in 20082011 in Jiangsu, and from 3.5

In recent years, there has been growing awareness that internal migration within China may be changing the distribution of the HIV epidemic by broadening social integration and connecting populations from different regions through sexual networks. ${ }^{1}$ 
Data from national behavioural surveillance reports from 2004 to 2005 showed that in nine mainland provinces, migrant HIV/AIDS cases accounted for more than $50 \%$ of total infections. ${ }^{5}$ A number of cohort studies on MSM conducted in China have revealed an HIV epidemic among MSM, but in most of these studies MSM retention was not very high. Retention rates for these cohort studies were rarely over $70 \%$ at 12 -month follow-up, with a few of them being below $20 \% .{ }^{6-9}$ The reasons for these low rates may include the migration of MSM. ${ }^{8}$ Moreover, studies conducted among urban MSM also revealed a high proportion of migrants among MSM in major cities such as Beijing $(88 \%),{ }^{10}$ Chongqing $(80.3 \%)^{11}$ and Shanghai $\left.(79.7 \%)\right)^{12}$ Although migration itself does not spread HIV or other sexually transmitted diseases (STDs), it may increase the possibility of infection when combined with high-risk behaviours, which are prevalent among MSM. It has been confirmed in numerous studies that high-risk behaviours such as unprotected anal sex, commercial sex, group sex, casual sex and having multiple sexual partners are prevalent among MSM in China. ${ }^{13-15}$ On the other hand, the perceived stigma and discrimination resulting from traditional Chinese culture and conservative social values have increasingly led Chinese MSM to marry female sexual partners, which leads to a potential risk of transmitting HIV to the general population via heterosexual transmission. ${ }^{14}$ As migrants lack the stability of a family or a home community and are hampered by generally low educational levels, limited knowledge of HIV/AIDS prevention, and discrimination and marginalisation from the mainstream metropolitan society, they may engage more frequently in high-risk behaviours. ${ }^{16} 17$ Furthermore, due to China's household registration system and urban social security system, migrants have limited access to comprehensive, convenient and longterm health services. ${ }^{18}$ Recently, some cross-sectional studies in China demonstrated that compared to local MSM, migrant MSM engage in more high-risk sexual behaviours such as having multiple sexual partners, higher frequencies of anal and oral intercourse, and unprotected sexual behaviours with both males and females. ${ }^{15} 19$ As a marginal and vulnerable subpopulation, migrant MSM should be accorded a high level of consideration by researchers.

Beijing, the capital of China and a centre of political, economic and cultural activity, is a magnet for migrants. In 2012, Beijing's metropolitan population included some 7.7 million migrants, with migrants accounting for $37.4 \%$ of the total population of Beijing (China National Bureau of Statistics, 2013). According to statistics from the Beijing Municipal Bureau of Health, the proportion of MSM among those with HIV/AIDS increased from $22.8 \%$ in 2006 to $69.1 \%$ at the end of October $2013 .{ }^{20}$ In addition, previous research among MSM in Beijing revealed that with the increasing epidemic of HIV among MSM in recent years, the number of migrants among recruited participants also showed a noticeable upward trend. ${ }^{10} 11$ This phenomenon implies the following: (1) migrant MSM play a major role in MSM society in Beijing; (2) this marginal population has emerged gradually with the constant improvement in sampling methods; and (3) studies more targeted on the particular population are urgently needed, since currently only a few cross-sectional studies have been conducted to investigate HIV/STD infections and relative risk factors in this group. ${ }^{21} 22$ Thus, the purpose of this research was to assess the incidence of HIV and syphilis and examine the predictors associated with seroconversion and retention in a prospective cohort study among migrant MSM in Beijing, China.

\section{METHODS}

\section{Study design and study population}

This prospective cohort study was conducted in Beijing. Participants were all Chinese citizens and were recruited through three methods. First, study participants were recruited directly through website advertisements by a non-governmental AIDS volunteer group (http://www. hivolunt.net). Second, peer recruiters were hired and trained to distribute flyers with study-related information at MSM-frequented venues (eg, MSM clubs, bars, parks and bathhouses). Third, study participants were encouraged to refer their peers for enrolment in the study. Once a potential participant had been referred or identified, local research team members approached the individual to verify eligibility, which included age $\geq 18$ years, male gender, HIV-negative, had sex with another male in the past 12 months, lacked permanent household registration in Beijing and provided written informed consent.

We calculated the sample size using the equation

$$
\mathrm{n}=\frac{\left(\mathrm{z}_{\alpha} \sqrt{2 \overline{\mathrm{pq}}}+\mathrm{z}_{\beta} \sqrt{\mathrm{p}_{0} \mathrm{q}_{0}+\mathrm{p}_{1} \mathrm{q}_{1}}\right)^{2}}{\left(\mathrm{p}_{1}-\mathrm{p}_{0}\right)^{2}},
$$

where $\alpha$ and $\beta$ are 0.05 and 0.10 and the corresponding $\mathrm{z}_{\alpha}$ and $\mathrm{z}_{\beta}$ are 1.96 and 1.282. $\mathrm{p}_{0}$ represents the prevalence of HIV among general MSM in Beijing, which was set at 0.02 according to the estimates of the HIV epidemic in 2012-2013 in Beijing; $p_{1}$ represents the HIV prevalence among migrant MSM in Beijing, which was estimated to be higher than $\mathrm{p}_{0}$ at 0.06 , so $\mathrm{n}$ was calculated to be 502. In case of loss to follow-up, we increased the target sample size by $10 \%$. A total of 547 participants were screened during the baseline screening survey from September to October 2009, of whom 36 were HIV-positive and 162 were syphilis-positive. In light of the curability and reinfection potential of syphilis, participants who were syphilis-positive at baseline were still recruited into the cohort. Thus, a total of 511 eligible and consenting MSM were enrolled into the prospective cohort, of whom 141 were syphilis-positive. Participants were followed up for 4 and 8 months after the baseline survey. At each study visit (baseline, 4-month follow-up 
and 8-month follow-up), eligible participants were interviewed by trained health professionals in a private room of the study clinic, and blood plasma specimens were collected and analysed for HIV and syphilis antibodies by experienced physicians. One week later, each participant received their test results anonymously through the use of a private identification code. All participants who tested positive for HIV/syphilis received additional post-test counselling and referrals to relevant free services. Every participant received 50 RMB (US\$7.4), 12 free condoms and one free lubricant after each completed study visit. Every participant was asked to provide at least two different and current methods of contact, and reminder calls were made before the follow-up day to verify that the exact time of the visit was convenient for them. The study protocol and informed consent forms were approved by the Institutional Review Boards of the National Center for AIDS/STD Control and Prevention of the China Center for Disease Control and Prevention (figure 1).

\section{Data collection and laboratory tests}

Questionnaire-based interviews were conducted on a one-on-one basis in a separate private room of the district clinic. Data on demographics, sexual behaviour and history of STDs were collected. Participants' questionnaires and blood specimens were linked through the assignment of a unique identifier code in the follow-up visits.

Blood specimens were tested for HIV and syphilis infection. HIV infection status was determined by ELISA (InTec Products, Xiamen, China) screening and confirmed by an HIV-1/2 Western blot (HIV Blot 2.2 WBTM, Genelabs Diagnostics, Singapore). Syphilis infection was determined by a rapid plasma reagin test (Shanghai Rongsheng, Shanghai, China) and confirmed using a Treponema pallidum particle agglutination assay (TPPA) test (Fujirebio, Tokyo, Japan).

\section{Statistical analysis}

Questionnaire data were double-entered and then checked for accuracy using EpiData software (V.3.1; The EpiData Association, Odense, Denmark). Data were analysed using Stata/SE (V.12.0 for Windows; StataCorp LP, College Station, USA). HIV seroconversion was estimated to have occurred at the midpoint between the time of the baseline HIV test and the time of the follow-up HIV test with a seropositive result. Syphilis
Figure 1 Study flowchart of the migrant men who have sex with men (MSM) cohort, with a focus on HIV and syphilis incidence.

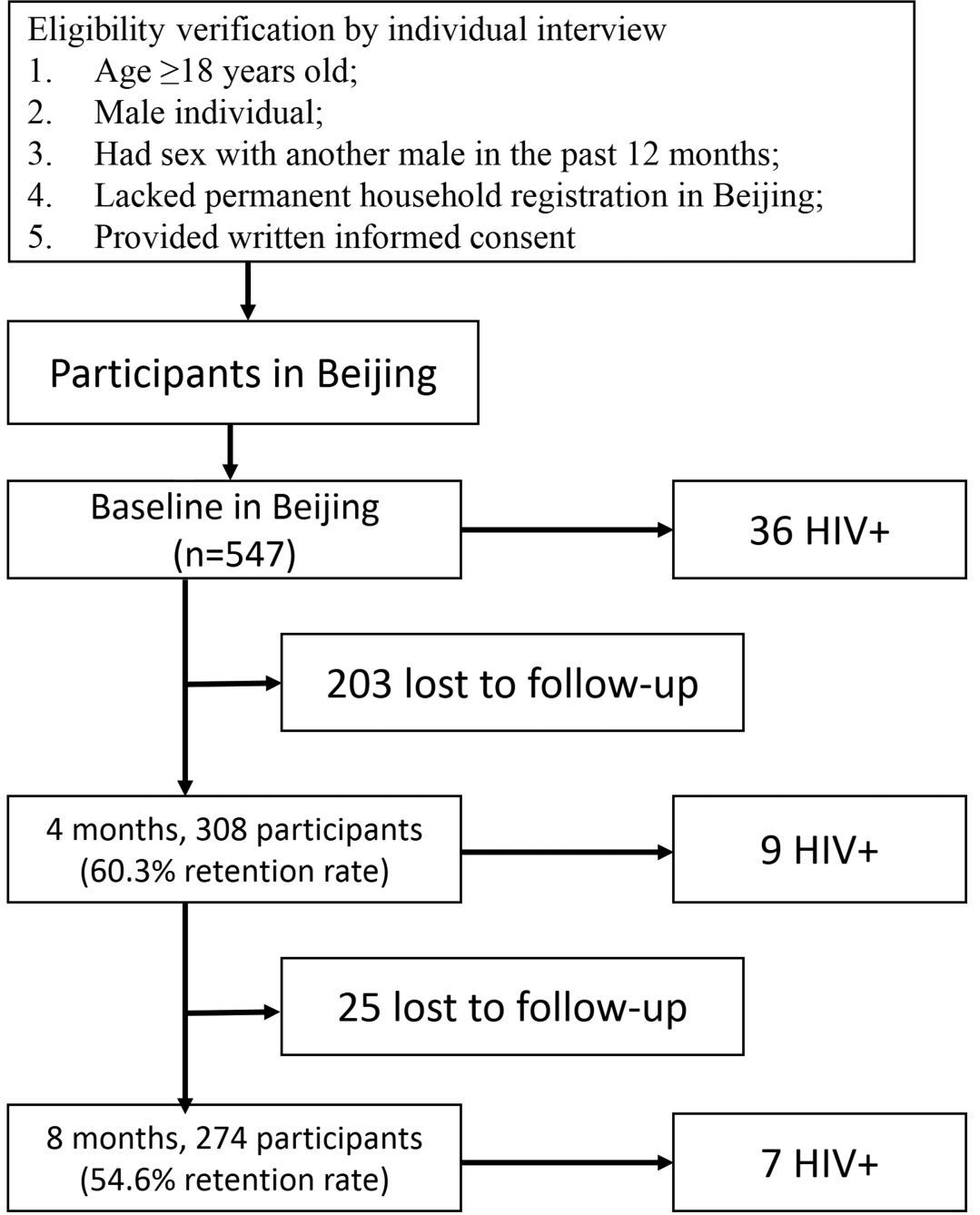


seroconversion was estimated to have occurred at the halfway point between the previous visit and the visit where seroconversion occurred. HIV/syphilis incidence density was calculated based on a Poisson distribution, with number of seroconversion incidents within the follow-up period as the numerator and PY over the entire follow-up period as the denominator. Categorical factors associated with retention rate were analysed by univariate logistic regression, including baseline demographic and behavioural characteristics. A stepwise multiple logistic regression (out 0.05 , in 0.02 ) was used to select independent factors for the retention rate. A Cox regression model was used in univariate and multiple regression analyses to identify correlates of time to HIV seroconversion. Statistically significant variables in univariate analysis $(p<0.10)$ were entered simultaneously into a multivariate Cox proportional hazard regression model to determine the adjusted HR (aHR) for HIV/ syphilis seroconversion-related risk factors.

\section{RESULTS}

\section{Baseline characteristics of the participants}

The median age of the 511 participants was 29 years, 94.32\% belonged to the Han ethnic group, $64.77 \%$ were single, $22.90 \%$ were married or cohabiting, and $12.33 \%$ were separated, divorced or widowed. As regards educational level, $73.19 \%$ had received a high school education or lower. About half $(54.01 \%)$ had monthly incomes of $<$ US\$300. Participants who identified themselves as exclusively homosexual or as bisexual accounted for $60.67 \%$ and $37.57 \%$ of the MSM, respectively. Overall, $16.05 \%$ and $44.03 \%$ of the participants thought it was impossible or improbable, respectively, that they would be infected with HIV. The median age of sexual debut was 20 years, and $58.71 \%$ experienced their sexual debut with a male. The median age of first anal intercourse was 22 , and $58.65 \%$ of participants did not use a condom at first anal intercourse. In the past year, $78.67 \%$ of the participants had ever received condoms, $65.17 \%$ had ever received lubricant, $23.68 \%$ had ever taken part in peer education, $18.40 \%$ had ever undergone examination or treatment for STDs and $52.49 \%$ had ever received voluntary counselling and testing services (VCTs).

As regards behaviours in the past 6 months among participants, the median number of male sex partners was three. Concerning high-risk behaviours, 327 $(63.99 \%)$ had oral sex with male sex partners, of whom $22(6.73 \%)$ consistently used a condom with regular sex partners, and $26(7.95 \%)$ with casual partners. All 511 $(100 \%)$ had anal sex with male sex partners, of whom $13.11 \%$ had consistently used a condom with regular sex partners, and $66(18.70 \%)$ with casual partners. A total of $61(11.94 \%)$ participants had ever engaged in commercial sexual behaviour, among whom 35 (57.38\%) had paid for sex and $43(70.49 \%)$ had received money for sex. A total of $59(11.55 \%)$ had ever engaged in group sex, which happened almost exclusively (96.61\%) among males. A total of 166 (32.49\%) had ever engaged in sex under the influence of alcohol, among whom 152 $(92.77 \%)$ had homosexual intercourse while intoxicated (table 1).

Incidence of HIV infection, syphilis infection, HIV-syphilis co-infection, and factors predicting HIV and syphilis seroconversion

During the 8-month follow-up, 16 HIV seroconversions were observed over 204.27 PY of observation, resulting in an incidence rate of 7.83 per $100 \mathrm{PY}$ (95\% CI 4.48 to 12.72). At the 4-month visit, the HIV incidence rate was 8.33 per 100 PY $(95 \%$ CI 3.81 to 15.81$)$, while at the 8-month visit it was 3.83 per 100 PY $(95 \%$ CI 1.54 to 7.89). During the 8-month follow-up period, the syphilis incidence rate was 11.11 per $100 \mathrm{PY}$ (95\% CI 6.47 to 17.80). At the 4- and 8-month visits, the syphilis incidence rates were 10.03 per 100 PY (95\% CI 4.33 to 19.76) and 15.25 per 100 PY (95\% CI 6.97 to 28.94), respectively. Six HIV seroconversion cases were observed, becoming syphilis-positive over 52.10 PY, resulting in an incidence of co-infection of 11.52 per 100 PY (95\% CI 4.23 to 25.07$)$.

Multivariate Cox regression analysis indicated that age $\leq 15$ years at first anal intercourse (aHR 9.20, 95\% CI 1.94 to $43.56, \mathrm{p}=0.050$ ) and ever had group sex (aHR $4.30,95 \%$ CI 1.40 to $13.18, \mathrm{p}=0.011$ ) were significantly associated with time to HIV seroconversion (table 2). Bisexual orientation (aHR 5.09, 95\% CI 1.02 to 25.57, $\mathrm{p}=0.048)$ and had more than one sex partners since last investigation (aHR 9.22, 95\% CI 1.13 to 73.37, $\mathrm{p}=0.038$ ) were significantly identified as risk factors for syphilis incidence (table 3).

\section{Predictors of cohort retention}

Of the 511 participants, $60.3 \%$ (308) were retained in the cohort at the 4-month follow-up and $54.6 \%$ (274) were retained at the 8-month follow-up.

Univariate factors among baseline characteristics which were significantly associated with 4-month retention were analysed with stepwise multiple logistic regression. Four factors were kept in the final model: age $>25$ years (adjusted OR (aOR) 1.81, 95\% CI 1.07 to 3.06, $\mathrm{p}=0.026$ ), living in Beijing for more than 1 year ( $\mathrm{aOR}$ $3.68,95 \%$ CI 2.04 to $6.64, \mathrm{p}<0.001)$, had at least one female sex partner since last investigation (aOR 0.48, 95\% CI 0.29 to $0.80, \mathrm{p}=0.005)$, and had ever received VCTs in the past 12 months (aOR 1.71, 95\% CI 1.07 to 2.72, $\mathrm{p}<0.024$ ) (table 4).

For 8-month retention, age $>25$ years (aOR 1.76, 95\% CI 1.16 to 2.68, $\mathrm{p}=0.008$ ), living in Beijing for more than 1 year (aOR $1.68,95 \%$ CI 1.03 to $2.73, \mathrm{p}=0.039$ ), first sexual partner was female (aOR $0.60,95 \%$ CI 0.41 to $0.88, \mathrm{p}=0.009)$, ever had group sex (aOR $0.50,95 \%$ CI 0.28 to $0.89, \mathrm{p}=0.019)$, and had received lubricant in the past 12 months (aOR 2.31, 95\% CI 1.56 to 3.42, 
Table 1 Sociodemographic characteristics of the 511 study participants in the cohort of migrant MSM in Beijing

\begin{tabular}{|c|c|c|}
\hline Variables & $\mathbf{n}$ & Per cent \\
\hline Overall, N & 511 & 100 \\
\hline \multicolumn{3}{|l|}{ Age } \\
\hline Median (years) & 29 & \\
\hline $18-24$ & 149 & 29.16 \\
\hline$\geq 25$ & 362 & 70.84 \\
\hline \multicolumn{3}{|l|}{ Ethnicity } \\
\hline Han & 482 & 94.32 \\
\hline Minority & 29 & 5.68 \\
\hline \multicolumn{3}{|l|}{ Marital status } \\
\hline Single & 331 & 64.77 \\
\hline Married & 114 & 22.31 \\
\hline Cohabiting with male or female sex partner & 3 & 0.59 \\
\hline Separated or divorced or widowed & 63 & 12.33 \\
\hline \multicolumn{3}{|l|}{ Duration of stay in Beijing } \\
\hline$<1$ year & 173 & 33.85 \\
\hline$\geq 1$ year & 338 & 66.14 \\
\hline \multicolumn{3}{|l|}{ Educational level } \\
\hline High school and below & 374 & 73.19 \\
\hline College and above & 137 & 26.81 \\
\hline \multicolumn{3}{|l|}{ Monthly income (US\$) } \\
\hline$<300$ & 276 & 54.01 \\
\hline$\geq 300$ & 235 & 45.99 \\
\hline \multicolumn{3}{|l|}{ Self-reported sexual orientation } \\
\hline Homosexual & 310 & 60.67 \\
\hline Heterosexual & 8 & 1.57 \\
\hline Bisexual & 192 & 37.57 \\
\hline Other & 1 & 0.2 \\
\hline \multicolumn{3}{|l|}{ Self-reported risk of HIV infection } \\
\hline Impossible & 82 & 16.05 \\
\hline Improbable & 225 & 44.03 \\
\hline Possible & 74 & 14.48 \\
\hline Probable & 15 & 2.94 \\
\hline No idea & 115 & 22.5 \\
\hline Age of sexual debut (median, years) & 20 & \\
\hline \multicolumn{3}{|l|}{ Gender of first sexual partner } \\
\hline Male & 300 & 58.71 \\
\hline Female & 211 & 41.29 \\
\hline Age of first anal intercourse (median, years) & 22 & \\
\hline Had first anal intercourse before 15 years of age & 13 & 2.54 \\
\hline Number of male sex partners in the last 6 months (median) & 3 & \\
\hline Number of female sex partners in the last 6 months (median) & 0 & \\
\hline Oral sex with regular partners with consistent condom use in the past 6 months & 22 & 6.73 \\
\hline Oral sex with casual partners with consistent condom use in the past 6 months & 26 & 7.95 \\
\hline Anal sex with regular partners with consistent condom use in the past 6 months & 67 & 13.11 \\
\hline Anal sex with casual partners with consistent condom use in the past 6 months & 66 & 18.7 \\
\hline Ever had commercial sexual behaviour & 61 & 11.94 \\
\hline Ever had group sex & 59 & 11.55 \\
\hline Alcohol use prior to sex & 166 & 32.49 \\
\hline Ever had STDs & 84 & 16.44 \\
\hline Have ever received condoms & 402 & 78.67 \\
\hline Have ever received lubricant & 333 & 65.17 \\
\hline Have ever received peer education & 121 & 23.68 \\
\hline Have ever received examination or treatment for STD & 94 & 18.4 \\
\hline Have ever received VCT services & 268 & 52.45 \\
\hline Have ever received educational materials on AIDS/STD & 302 & 59.1 \\
\hline
\end{tabular}


Table 2 Multivariate analyses of participant characteristics associated with incident HIV infection among migrant MSM in Beijing

\begin{tabular}{|c|c|c|c|c|c|c|c|}
\hline \multirow[b]{2}{*}{ Factors } & \multirow[b]{2}{*}{$\begin{array}{l}\text { No. of HIV } \\
\text { seroconversions }\end{array}$} & \multirow[b]{2}{*}{$\begin{array}{l}\text { Cumulative } \\
\text { PY }\end{array}$} & \multirow{2}{*}{$\begin{array}{l}\text { HIV } \\
\text { incidence } \\
\text { (/100 PY) }\end{array}$} & \multicolumn{2}{|l|}{ Univariate } & \multicolumn{2}{|l|}{ Multivariate } \\
\hline & & & & HR (95\% Cl) & $\begin{array}{l}\mathbf{p} \\
\text { Value }\end{array}$ & $\begin{array}{l}\text { Adjusted HR } \\
(95 \% \mathrm{Cl})\end{array}$ & $\begin{array}{l}\mathbf{p} \\
\text { Value }\end{array}$ \\
\hline Total & 16 & 204.27 & 7.83 & & & & \\
\hline \multicolumn{8}{|l|}{ Education } \\
\hline $\begin{array}{l}\text { Junior college } \\
\text { and below }\end{array}$ & 13 & 143.95 & 9.03 & 1.00 & & & \\
\hline $\begin{array}{l}\text { Above junior } \\
\text { college }\end{array}$ & 3 & 60.32 & 4.97 & $0.56(0.16$ to 1.98$)$ & 0.370 & & \\
\hline \multicolumn{8}{|c|}{ Seeking partner through internet } \\
\hline No & 12 & 109.45 & 10.96 & 1.00 & & & \\
\hline Yes & 4 & 94.81 & 4.22 & $0.39(0.12$ to 1.20$)$ & 0.100 & & \\
\hline \multicolumn{8}{|c|}{ Age of first anal intercourse (median, years) } \\
\hline$\geq 15$ & 14 & 200.99 & 6.97 & 1.00 & & 1.00 & \\
\hline$<15$ & 2 & 3.28 & 60.98 & 8.28 (1.88 to 36.46$)$ & 0.005 & 9.20 (1.94 to 43.56$)$ & 0.005 \\
\hline \multicolumn{8}{|c|}{ Ever had group sex } \\
\hline No & 11 & 183.48 & 6 & 1.00 & & 1.00 & \\
\hline Yes & 5 & 20.78 & 24.06 & 3.95 (1.37 to 11.38$)$ & 0.011 & $4.30(1.40$ to 13.18$)$ & 0.011 \\
\hline \multicolumn{8}{|c|}{ Knowledge of HIV/AIDS was acquired from } \\
\hline \multicolumn{8}{|c|}{ Media } \\
\hline No & 12 & 109.65 & 10.94 & 1.00 & & & \\
\hline Yes & 4 & 94.62 & 4.23 & $0.39(0.12$ to 1.20$)$ & 0.100 & & \\
\hline \multicolumn{8}{|l|}{ Internet } \\
\hline No & 6 & 108.59 & 5.53 & 1.00 & & & \\
\hline Yes & 10 & 95.68 & 10.45 & $0.38(0.12$ to 1.19$)$ & 0.097 & & \\
\hline \multicolumn{8}{|c|}{ Consistent condom use during oral sex with regular partners since last investigation } \\
\hline No & 1 & 9.45 & & 1.00 & & & \\
\hline Yes & 15 & 194.82 & & $0.73(0.10$ to 5.50$)$ & 0.758 & & \\
\hline \multicolumn{8}{|c|}{ Consistent condom use during oral sex with casual partners since last investigation } \\
\hline No & 0 & 7.70 & 0 & 1.00 & & & \\
\hline Yes & 9 & 128.67 & 6.99 & - & - & & \\
\hline \multicolumn{8}{|c|}{ Consistent condom use during anal sex with regular partners since last investigation } \\
\hline No & 3 & 29.18 & 10.28 & 1.00 & & & \\
\hline Yes & 13 & 175.09 & 7.42 & $0.72(0.21$ to 2.53$)$ & 0.609 & & \\
\hline \multicolumn{8}{|c|}{ Consistent condom use during anal sex with casual partners since last investigation } \\
\hline No & 2 & 27.56 & 7.26 & 1.00 & & & \\
\hline Yes & 10 & 115.99 & 8.62 & 1.19 (0.26 to 5.42$)$ & 0.825 & & \\
\hline
\end{tabular}

$\mathrm{p}<0.001)$ were retained in the final model using stepwise multiple logistic regression analysis (table 5).

\section{DISCUSSION}

Our study found that the prevalence of HIV (6.58\%) among migrant MSM was at epidemic levels, which was remarkable when compared to that of a previous study among the general migrant population $(0 \%)$ in Beijing. ${ }^{23}$ Additionally, it was higher than the $4.8 \%$ in 2007 among general MSM in Beijing. ${ }^{24} \mathrm{~A}$ similar result $(5.9 \%)$ was found in a recent cross-sectional study among migrant MSM. $^{23}$ Incidence in this subgroup $(7.83 / 100 \mathrm{PY})$ was consistent with that of migrant MSM (8.51/100 PY) in an MSM cohort study in Beijing, and higher than the incidence among permanent resident MSM. ${ }^{25}$ It was also higher than the incidence of general MSM in different parts of China. ${ }^{6} 7$
The study found that participants whose first anal intercourse occurred before age 15 had significantly higher risk for HIV seroconversion. An American study of young minority MSM found that study participants who had an MSM sexual debut before 16 years of age were more likely to have psychological problems and high-risk behaviours like exchanging sex and drug use. ${ }^{26}$ Early sexual debut could have a deep and long-term influence on individuals' attitudes towards sex, but appropriate sex education has generally been shunned in traditional Chinese education. Our study indicated that early sex education should be adapted to and take account of Chinese tradition, and that relevant measures need to be implemented as soon as possible. Another risk factor significantly associated with HIV incidence was ever had group sex. An explanation of this observation is that psychological stress caused by stigma and discrimination hinders the development of longer term 
Table 3 Multivariate analyses of participant characteristics associated with incident syphilis infection among migrant MSM in Beijing

\begin{tabular}{|c|c|c|c|c|c|c|c|}
\hline \multirow[b]{2}{*}{ Factors } & \multirow[b]{2}{*}{$\begin{array}{l}\text { No. of syphilis } \\
\text { seroconversions }\end{array}$} & \multirow[b]{2}{*}{$\begin{array}{l}\text { Cumulative } \\
\text { PY }\end{array}$} & \multirow{2}{*}{$\begin{array}{l}\text { Syphilis } \\
\text { incidence } \\
\text { (/100 PY) }\end{array}$} & \multicolumn{2}{|l|}{ Univariate } & \multicolumn{2}{|l|}{ Multivariate } \\
\hline & & & & $\mathrm{HR}(95 \% \mathrm{Cl})$ & p Value & $\begin{array}{l}\text { Adjusted HR } \\
(95 \% \mathrm{Cl})\end{array}$ & p Value \\
\hline Total & 17 & 152.95 & 11.11 & & & & \\
\hline \multicolumn{8}{|c|}{ Ever had female sex partner(s) } \\
\hline No & 9 & 96.89 & 9.29 & 1.00 & & & \\
\hline Yes & 8 & 54.64 & 14.64 & $5.56(1.10$ to 27.05$)$ & 0.038 & 5.09 (1.02 to 25.57$)$ & 0.048 \\
\hline Other & 0 & 0 & - & - & & & \\
\hline \multicolumn{8}{|c|}{ Number of female sex partners since last investigation } \\
\hline$\leq 1$ & 16 & 149.9 & 10.67 & 1.00 & & & \\
\hline$\geq 2$ & 1 & 3.05 & 32.79 & $9.22(1.13$ to 75.37$)$ & 0.038 & & \\
\hline \multicolumn{8}{|c|}{ Seeking partners in bar/dancing hall/tearoom/saloon } \\
\hline No & 11 & 129.41 & 8.5 & 1.00 & & & \\
\hline Yes & 6 & 23.55 & 25.48 & $3.12(0.75$ to 13.07$)$ & 0.119 & & \\
\hline \multicolumn{8}{|c|}{ Consistent condom use during oral sex with regular partners in the past 6 months } \\
\hline No & 1 & 6.24 & 16.03 & 1.00 & & & \\
\hline Yes & 16 & 146.71 & 10.91 & $0.24(0.03$ to 1.99$)$ & 0.188 & & \\
\hline \multicolumn{8}{|c|}{ Consistent condom use during oral sex with casual partners in the past 6 months } \\
\hline No & 0 & 4.37 & 0 & 1.00 & & & \\
\hline Yes & 8 & 92.58 & 8.64 & - & & & \\
\hline \multicolumn{8}{|c|}{ Consistent condom use during anal sex with regular partners in the past 6 months } \\
\hline No & 2 & 24.52 & 8.16 & 1.00 & & & \\
\hline Yes & 15 & 128.43 & 11.68 & $0.57(0.11$ to 2.80$)$ & 0.485 & & \\
\hline \multicolumn{8}{|c|}{ Consistent condom use during anal sex with casual partners in the past 6 months } \\
\hline No & 0 & 19.80 & 0 & 1.00 & & & \\
\hline Yes & 11 & 83.21 & 13.22 & - & & & \\
\hline
\end{tabular}

relationships between $\mathrm{MSM}^{27}$ and having sex with multiple partners increases the chance of HIV transmission. This indicates a need for confidentiality and supportive services for migrant MSM. Serious attention should also be paid to the prevalence of STDs. The prevalence of syphilis $(29.62 \%)$ was higher than in previous studies conducted among MSM in Beijing, which found a prevalence of $19.8 \%$ in $2007^{24}$ and $22.0 \%$ in $2009 .{ }^{10}$ The incidence of syphilis $(11.11 / 100 \mathrm{PY})$ was also extremely high compared to the rate of $8.06 / 100 \mathrm{PY}$ reported in a cohort study among MSM during the same period in $2009 .^{25}$ A high prevalence of syphilis among MSM is a common risk factor for HIV infection because of the biological and behavioural links between syphilis and HIV. $^{28}$

The multiple regression analysis significantly revealed that self-identified bisexuals were five times more likely than homosexuals to be infected with syphilis. One

Table 4 Factors associated with 4-month retention rate in a cohort study of migrant MSM in Beijing, China, using stepwise multiple logistic analysis

\begin{tabular}{|c|c|c|c|c|c|c|}
\hline Factor & $\mathbf{N}$ & Retention rate \% (n) & OR $(95 \% \mathrm{Cl})$ & p Value & Adjusted OR (95\% Cl) & p Value \\
\hline \multicolumn{7}{|l|}{ Age } \\
\hline $18-24$ & 149 & $46.98(70)$ & 1.00 & & 1.00 & \\
\hline$\geq 25$ & 362 & $65.75(238)$ & $2.17(1.47$ to 3.19$)$ & $<0.001$ & $1.81(1.07$ to 3.06$)$ & 0.026 \\
\hline \multicolumn{7}{|c|}{ Duration of stay in Beijing } \\
\hline$<1$ year & 95 & $34.74(33)$ & 1.00 & & 1.00 & \\
\hline$\geq 1$ year & 416 & $66.27(208)$ & 3.66 (2.29 to 5.85$)$ & $<0.001$ & 3.68 (2.04 to 6.64$)$ & $<0.001$ \\
\hline \multicolumn{7}{|c|}{ Number of female sex partners since last investigation } \\
\hline 0 & 382 & $64.66(247)$ & 1.00 & & 1.00 & \\
\hline$\geq 1$ & 129 & $47.29(61)$ & $0.49(0.33$ to 0.74$)$ & 0.001 & $0.48(0.29$ to 0.80$)$ & 0.005 \\
\hline \multicolumn{7}{|c|}{ Have received VCT services in the past 12 months } \\
\hline No & 243 & $51.85(126)$ & 1.00 & & 1.00 & \\
\hline Yes & 268 & $67.91(182)$ & $1.97(1.37$ to 2.81$)$ & $<0.001$ & $1.71(1.07$ to 2.72$)$ & 0.024 \\
\hline
\end{tabular}


Table 5 Factors associated with 8-month retention rate in a cohort study of migrant MSM in Beijing, China, using stepwise multiple logistic analysis

\begin{tabular}{|c|c|c|c|c|c|c|}
\hline Factor & $\mathbf{N}$ & Retention rate \% (n) & OR $(95 \% \mathrm{Cl})$ & p Value & Adjusted OR (95\% CI) & p Value \\
\hline \multicolumn{7}{|l|}{ Age } \\
\hline $18-24$ & 149 & $44.30(66)$ & 1.00 & & 1.00 & \\
\hline$\geq 25$ & 362 & $57.18(207)$ & 1.68 (1.14 to 2.47$)$ & 0.008 & 1.76 (1.16 to 2.68$)$ & 0.008 \\
\hline \multicolumn{7}{|c|}{ Duration of stay in Beijing } \\
\hline$<1$ year & 78 & $48.72(38)$ & 1.00 & & 1.00 & \\
\hline$>1$ year & 338 & $58.88(199)$ & 2.17 (1.37 to 3.43$)$ & $<0.001$ & 1.68 (1.03 to 2.73$)$ & 0.039 \\
\hline \multicolumn{7}{|c|}{ Gender of first sexual partner } \\
\hline Male & 300 & $58.33(175)$ & 1.00 & & 1.00 & \\
\hline Female & 211 & $46.45(98)$ & $0.62(0.43$ to 0.88$)$ & 0.008 & $0.60(0.41$ to 0.88$)$ & 0.009 \\
\hline \multicolumn{7}{|c|}{ Ever had group sex } \\
\hline No & 452 & $54.87(248)$ & 1.00 & & 1.00 & \\
\hline Yes & 59 & $42.37(25)$ & $0.61(0.35$ to 1.05$)$ & 0.072 & $0.50(0.28$ to 0.89$)$ & 0.019 \\
\hline \multicolumn{7}{|c|}{ Have received lubricant in the past 12 months } \\
\hline No & 109 & $34.86(38)$ & 1.00 & & 1.00 & \\
\hline Yes & 402 & $58.46(235)$ & 2.59 (1.78 to 3.77$)$ & $<0.001$ & 2.31 (1.56 to 3.42$)$ & $<0.001$ \\
\hline
\end{tabular}

major reason for this finding could be that MSM who had had sex with females may have more varied sexual behaviours with a wider range of sexual partners, including males and females. In addition, a cross-sectional study among young migrant MSM in Beijing revealed that MSM who were engaged in bisexual behaviour had a higher rate of unprotected sex with stable female partners and were less likely to take part in preventive behaviours, ${ }^{18}$ which was consistent with our finding that those who had at least one female sex partner and those whose first sexual partner was female were significantly more likely to be lost at the 4- and 8-month follow-up visits, respectively. MSM face strong social pressure and stigma in China, which may lead them to hide their sexual orientation by unwillingly engaging in heterosexual relationships; thus, many Chinese MSM will potentially enter a heterosexual marriage due to social and familial pressure. ${ }^{4}$ Concerned about disclosing their MSM behaviour to their female partners, bisexual MSM may be more reluctant to be followed up. Also, MSM with female partners are more likely to be engaged in risk behaviours, such as having unprotected sex to demonstrate loyalty to their female partners and having commercial or casual sex with men. Moreover, only $18.4 \%$ of the participants reported having undergone an examination or treatment for STDs in the past 12 months, which may be related to the high cost of health services and low coverage of health insurance for the migrant population. ${ }^{18}$ Low risk-awareness might be another reason for their lower access to HIV/STD testing and treatment. All of these factors emphasise the urgent need for widespread and accurate syphilis screening and affordable treatment for migrant MSM in Beijing.

In our study, the retention rate was lower than in previous cohort studies conducted among general MSM in Beijing (86.2\% in 2007, 86.8\% in 2009). ${ }^{25} 26$ The multiple regression analysis showed that participants who were less than 25 years old were more likely to be lost at both the 4 - and 8-month follow-up visits. Similar problems have occurred among studies of young MSM in the USA. ${ }^{29}$ A plausible reason for this finding was that many young people in China have poor knowledge of safe sexual behaviours. Traditional interventions are more pragmatic but less attractive and appropriate for young people. A qualitative investigation conducted among young MSM in Milwaukee and Detroit in the USA offered some new approaches, such as integrating HIV/AIDS care intervention into the daily lives of participants and helping solve social relationship problems. ${ }^{30}$ However, as young Chinese MSM most likely differ from Americans in some aspects, intervention measures among young MSM in Beijing deserve further investigation.

Regarding positive predictors, we found those who had been in Beijing for more than 1 year or who had received lubricant were more likely to be retained in this cohort at both the 4- and 8-month follow-up visits. A similar result was found in Yangzhou. ${ }^{8}$ Migrant MSM who stay longer in Beijing may have more chances to receive effective and stable intervention services, and consequent increasing awareness of self-protection might encourage them to pay closer attention to advances in research. As a result, migrants may be more likely to remain in the study to demonstrate their support. However, the data revealed that receiving VCT services in the past 12 months was a positive factor at the 4-month follow-up visit, which was replaced by receiving lubricant at the 8-month visit. This indicates that factors associated with retention may change as time goes on, which implies that we should adjust intervention methods over time so as to keep the entire cohort more stable. The results of our study showed that the provision of interventions for this population was limited. A specific intervention strategy targeted at migrant MSM and 
the mobility problem inherent to migrant populations is urgently needed.

This study has a number of limitations. First, retention rates were relatively low, which means that the estimates of $\mathrm{HIV} /$ syphilis seroconversion may be biased because we do not know whether the characteristics of the participants retained in the study were the same as those of the participants lost to follow-up. Second, the use of non-random sampling methods may have led to selection bias. Third, the sample size was insufficient, and some potentially relevant factors may be concealed by the small sample size. Last, the study subjects were all immigrants to and not emigrants from Beijing, and the proportion of migrants from different provinces varied. There is no evidence to show the data could be generalised to other regions.

To our knowledge, this is the first cohort study to assess the incidence of HIV and syphilis among migrant MSM in Beijing. With extremely prevalent risk behaviours, the migrant MSM had a high level of HIV incidence, and a serious epidemic of syphilis was found among this subgroup. Bisexual MSM were at a high risk of syphilis seroconversion but were less visible among migrant MSM. Future prevention efforts should include screening and appropriate treatment for syphilis. Different types of intervention services need to be implemented for this marginalised group. To improve the health of bisexual MSM and that of their partners, more attention should be paid to the female partners of bisexual MSM to encourage them to undergo testing and follow up. Risk behaviours and the HIV/STD epidemic among migrant MSM need to be investigated in the future to facilitate greater understanding.

\section{Author affiliations}

${ }^{1}$ Department of Epidemiology and Biostatistics, School of Public Health, Nanjing Medical University, Nanjing, Jiangsu, China

${ }^{2}$ Department of STDs/AIDS Prevention and Control, Beijing Center for Disease Prevention and Control, Beijing, China

${ }^{3}$ National Center for AIDS/STD Control and Prevention, Chinese Center for Disease Control and Prevention, Beijing, China

Contributors ZP, RY and NW conceived and designed the experiments. HL and $L W$ performed the experiments. HM and WM analysed the data. $\mathrm{HZ}$ and YZ contributed reagents, materials and/or analysis tools. HM, WM and ZP wrote the paper, and all authors read and approved the final version of the manuscript.

Funding This work was supported in part by the Natural Science Foundation of China $(81001288,11171268)$, the National S\&T Major Project Foundation of China (No. 2012ZX10001-001 and No. 2011ZX10004-902), and the Priority Academic Program Development of Jiangsu Higher Education Institutions (PAPD).

Competing interests None.

Provenance and peer review Not commissioned; externally peer reviewed.

Data sharing statement No additional data are available.

Open Access This is an Open Access article distributed in accordance with the Creative Commons Attribution Non Commercial (CC BY-NC 4.0) license, which permits others to distribute, remix, adapt, build upon this work noncommercially, and license their derivative works on different terms, provided the original work is properly cited and the use is non-commercial. See: http:// creativecommons.org/licenses/by-nc/4.0/

\section{REFERENCES}

1. Jia Z, Wang L, Chen RY, et al. Tracking the evolution of HIV/AIDS in China from 1989-2009 to inform future prevention and control efforts. PLoS One 2011;6:e25671.

2. National Center for STD/AIDS Prevention and Control, Chinese Center for Disease Prevention and Control. Epidemic of HIVIAIDS and sexual transmitted diseases and main progression on prevention in 2011, China. Beijing: Chinese Center for Disease Control and Prevention, 2012

3. Ministry of Health of the People's Republic of China, UNAIDS, WHO National report for HIVIAIDS estimation in China, 2011. Beijing: Chinese Center for Disease Control and Prevention, 2011.

4. Chow EPF, Lau JTF, Zhuang X, et al. HIV prevalence trends, risky behaviours, and governmental and community responses to the epidemic among men who have sex with men in China. Biomed Res Int 2014;2014:607261.

5. Lu F, Fu J, Zhang R, et al. Analysis of HIV epidemic situation and immigrant population. Infect Dis Info 2006;19:259-61.

6. Xu J, An M, Han X, et al. Prospective cohort study of HIV incidence and molecular characteristics of HIV among men who have sex with men (MSM) in Yunnan Province, China. BMC Infect Dis 2013;13:3.

7. Xu J, Zhang M, Brown K, et al. Syphilis and HIV seroconversion among a 12-month prospective cohort of men who have sex with men in Shenyang, China. Sex Transm Dis 2010;37:432-9.

8. Peng Z, Yang H, Norris J, et al. HIV incidence and predictors associated with retention in a cohort of men who have sex with men in Yangzhou, Jiangsu Province, China. PloS One 2012;7: e52731.

9. Yang $\mathrm{H}$, Tang $\mathrm{W}$, Xiao $\mathrm{Z}$, et al. Worsening epidemic of HIV and syphilis among men who have sex with men in Jiangsu Province, China. Clin Infect Dis 2014;58:1753-9.

10. Fan S, Lu H, Ma X, et al. Behavioral and serologic survey of men who have sex with men in Beijing, China: implication for HIV intervention. AIDS Patient Care STDS 2012;26:148-55.

11. Feng L, Ding X, Lu R, et al. High HIV prevalence detected in 2006 and 2007 among men who have sex with men in China's largest municipality: an alarming epidemic in Chongqing, China. J Acquir Immune Defic Syndr 2009;52:79-85.

12. Berg CJ, Nehl EJ, Wong FY, et al. Prevalence and correlates of tobacco use among a sample of MSM in Shanghai, China. Nicotine Tob Res 2011;13:22-8.

13. Liu $\mathrm{H}$, Yang $\mathrm{H}$, Li $\mathrm{X}$, et al. Men who have sex with men and human immunodeficiency virus/sexually transmitted disease control in China. Cell Res 2005;15:858.

14. Lau JT, Wang M, Wong HN, et al. Prevalence of bisexual behaviors among men who have sex with men (MSM) in China and associations between condom use in MSM and heterosexual behaviors. Sex Transm Dis 2008;35:406-13.

15. He N, Wong FY, Huang ZJ, et al. HIV risks among two types of male migrants in Shanghai, China: money boys vs. general male migrants. AIDS 2007;21:S73-9.

16. Yang X, Derlega VJ, Luo H. Migration, behaviour change and HIV/ STD risks in China. AIDS Care 2007;19:282-8.

17. Yang $X$. Temporary migration and HIV risk behaviors in China. Environ Plan A 2006;38:1527.

18. Peng $\mathrm{Y}$, Chang $\mathrm{W}, \mathrm{Zhou} \mathrm{H}$, et al. Factors associated with health-seeking behavior among migrant workers in Beijing, China. BMC Health Serv Res 2010;10:69.

19. Shi TX, Zhang BC, Li XF, et al. Study on the high risk behaviors related to AIDS among men who having sex with men in the floating population. Chin J Epidemiol 2009;30:668.

20. Newly reported HIV/AIDS cases increase $24.3 \%$ per year in Beijing. Retrieved from http://health.sina.com.cn/news/2013-11-29/ 0921114941.shtml (accessed 31 May 2014).

21. Wang B, Li X, Stanton B, et al. Socio-demographic and behavioral correlates for HIV and syphilis infections among migrant men who have sex with men in Beijing, China. AIDS Care 2013;25:249-57.

22. Guo Y, Li X, Song Y, et al. Bisexual behavior among Chinese young migrant men who have sex with men: implications for HIV prevention and intervention. AIDS Care 2012;24:451-8.

23. Xia DY, Lu HY, Liu GW, et al. Prevalence of HIV infection, syphilis, $\mathrm{HCV}$ and risk behaviors among migrant population in Beijing. Chin $J$ AIDS STD 2011;17:430-3.

24. Ruan $Y$, Luo F, Jia $Y$, et al. Risk factors for syphilis and prevalence of HIV, hepatitis $B$ and $C$ among men who have sex with men in Beijing, China: implications for HIV prevention. AIDS Behav 2009;13:663-70.

25. Li D, Li S, Liu Y, et al. HIV incidence among men who have sex with men in Beijing: a prospective cohort study. BMJ Open 2012;2: e001829. 
26. Outlaw AY, Phillips G, Hightow-Weidman LB, et al. Age of MSM sexual debut and risk factors: results from a multisite study of racial/ ethnic minority YMSM living with HIV. AIDS Patient Care STDS 2011;25:S23-9.

27. Neilands TB, Steward WT, Choi KH. Assessment of stigma towards homosexuality in China: a study of men who have sex with men Arch Sex Behav 2008;37:838-44.

28. Guo H, Wei JF, Yang H, et al. Rapidly increasing prevalence of HIV and syphilis and HIV-1 subtype characterization among men who have sex with men in Jiangsu, China. Sex Transm Dis 2009;36:120-5.

29. Galvin SR, Cohen MS. The role of sexually transmitted diseases in HIV transmission. Nat Rev Microbiol 2004;2:33-42.

30. Hightow-Weidman LB, Jones K, Wohl AR, et al. Early linkage and retention in care: findings from the outreach, linkage, and retention in care initiative among young men of color who have sex with men. AIDS Patient Care STDS 2011;25(Suppl 1): S31-8. 